\title{
Cohomologies of Lie Algebras of Formal Vector Fields Preserving a Foliation
}

\author{
By
}

\section{Atsumi HAMASAKI*}

\section{§ 0 . Introduction}

Let $a_{n}$ be the topological Lie algebra of all formal vector fields on $\boldsymbol{R}^{n}$ with the Krull topology; i. e.

$$
\mathfrak{a}_{n}=\left\{\sum_{i=1}^{n} f_{\imath}\left(x_{1}, \cdots, x_{n}\right) \partial / \partial x_{\imath} \mid f_{i} \in \boldsymbol{R}\left[\left[x_{1}, \cdots, x_{n}\right]\right]\right\}
$$

where $\mathbb{R}\left[\left[x_{1}, \cdots, x_{n}\right]\right]$ is the ring of formal power series in $n$ variables. In [3] I. M. Gel'fand and D. B. Fuks have calculated the entire cohomology of $\mathfrak{a}_{n}$. In this paper we shall study the following subalgebra of $a_{n}$ :

$$
\begin{aligned}
\mathfrak{a}_{r, n-r}= & \left\{\sum_{i=1}^{r} f_{i}\left(x_{1}, \cdots, x_{r}\right) \partial / \partial x_{i}\right. \\
& +\sum_{j=1}^{n-r} g_{J}\left(x_{1}, \cdots, x_{r}, y_{1}, \cdots, y_{n-r}\right) \partial / \partial y_{j} \mid \\
& \left.f_{\imath} \in \mathbb{R}\left[\left[x_{1}, \cdots, x_{r}\right]\right], g, \in \boldsymbol{R}\left[\left[x_{1}, \cdots, x_{r}, y_{1}, \cdots, y_{n-r}\right]\right]\right\} .
\end{aligned}
$$

The cohomology of this subalgebra was first studied by B. L. Feigin in [2] in order to construct the characteristic classes of flags of foliations. In the same paper the entire cohomology of $a_{1, n-1}$ was calculated by using a result about a cohomology with nontrivial coefficients (cf. [4]). Concerning a more general case A. Haefliger questioned whether

$$
H^{i}\left(\mathfrak{a}_{n, r}, \boldsymbol{R}\right) \cong H^{i}\left(\mathfrak{a}_{r}, \boldsymbol{R}\right) \text { for } i \leqq 2 n \text { (canonically). }
$$

In [12] K. Sithanantham proved this isomorphism for $i \leqq n-r$ by adopting the method of [13]. In this paper we prove this isomorphism for $i \leqq n+r$ using the tool which they employed and the result obtained in [7]. The Main theorem of this paper is the following :

Communicated by N. Shimada, January 25, 1988.

* Research Institute for Mathematical Sciences, Kyoto University, Kyoto 606, Japan. 
Theorem. Let $\iota: \mathfrak{a}_{r} \longrightarrow \mathfrak{a}_{r, n-r}$ be the natural inclusion. Then c induces an isomorphism of cohomology

$$
\iota^{*}: H^{i}\left(\mathfrak{a}_{r, n-r} ; \boldsymbol{R}\right) \longrightarrow H^{i}\left(\mathfrak{a}_{r} ; \boldsymbol{R}\right) \text { for } i \leqq n+r .
$$

It is known that $H^{*}\left(\mathfrak{a}_{r} ; \boldsymbol{R}\right)$ is $2 \mathrm{r}-$ connected ([3]).

Corollary.

$$
H^{i}\left(\mathfrak{a}_{r, n-r} ; \boldsymbol{R}\right)=0 \quad \text { for } i \leqq 2 r,
$$

and

$$
H^{2 r+1}\left(a_{r, n-r} ; \boldsymbol{R}\right) \neq 0 .
$$

This paper consists of 5 sections. In $\S 1$, we prove a key proposition which is a useful tool to calculate the cohomology of the classical infinite dimensional Lie algebras. In $\S 2$, we recall the definition of the Weil algebra and a spectral sequence converging to it. In $\S 3$, we make the theorem obtained in [7] appropriate to the general infinite dimensional case. In $\S 4$, we shall prove the main theorem. In $\S 5$, we give a result and a conjecture concerning the Weil algebra of an infinite dimensional Lie algebra.

The author would like to express his gratitude to professors $\mathrm{N}$. Shimada and M.Adachi for their encouragement and helpful suggestions.

\section{§1. Proof of a Key Proposition}

In this section we prove a key proposition which plays an important role in calculating the stable cohomology of transitive infinite Lie subalgebras of $\mathfrak{a}_{n}$.

First we recall a definition and notations. Let $k$ be a commutative field of characteristic zero. In this section cochain complexes and algebras are defined over $k$.

Definition 1.1. An operation of a Lie algebra $g$ in a cochain complex $\left\{C^{q}, d\right\}_{q=0,1,2}$, is a pair $(\ell, \theta)$ where :

(i) $\theta$ is a representation of $g$ in the graded module $C^{*}$, homogeneous degree zero.

(ii) $c$ is a linear map of $g$ to the space of endomorphisms of 
$C^{*}$, such that each $\iota(X)(X \in \mathrm{g})$ is homogeneous of degree -1 .

(iii) The following relations hold:

$$
\begin{aligned}
\theta(X) & =\iota(X) d+d \iota(X) & X \in \mathfrak{g} \\
\iota([X, Y]) & =\theta(X) \iota(X)-\iota(X) \theta(X) & X, Y \in \mathfrak{g} .
\end{aligned}
$$

When there is given an operation of a Lie algebra $g$ in a cochain complex $C^{*}$, we say that $C^{*}$ is a $\mathrm{g}$-cochain complex. The subcomplex of $C^{*}$ consisting of $\mathrm{g}$-invariant elements annihilated by $\iota(X)$ for all $X \in \mathrm{g}$ is called the basic subcomplex of $C^{*}$, denoted by $C_{\mathbb{B}^{-b a s i c}}^{*}$ or $C_{\text {basic }}^{*}$.

Next we consider a special case;i.e. an operation of a finite dimensional abelian Lie algebra $T$. It is well-known that any representation of Lie algebra $T$ can be extended to an action of the universal enveloping algebra of $T$, which is denoted by $U(T)$. Now we state the key proposition and prove it (cf. [11]).

Proposition 1.3. Let $T$ be a finite dimensional abelian Lie algebra and $\left\{C^{q}, d\right\}_{q=0,1,2, \ldots}$ be a $T$-cochain complex. Then if each $C^{q}$ is a projective $U(T)$-module, we have

$$
H^{i}(C)=0 \quad \text { for } i<\operatorname{dim} T .
$$

Proof. Since $T$ is abelian, $U(T)$ is isomorphic to a polynomial algebra. Hence we can consider the Koszul resolution (cf. [9, p 204]) :

$$
0 \longrightarrow \wedge^{n} T \otimes U(T) \stackrel{\delta}{\longrightarrow} \wedge^{n-1} T \otimes U(T) \stackrel{\delta}{\longrightarrow} \cdots \stackrel{\delta}{\longrightarrow} U(T) \stackrel{\varepsilon}{\longrightarrow} k \longrightarrow 0
$$

where $n=\operatorname{dim} T$ and $\wedge^{p}$ is the $p$-th exterior product of $T$. Since both operators $\delta$ and $d$ are commutative with action of $U(T)$, we can define the following double complex :

$$
\begin{aligned}
A & =\left\{(\wedge T \otimes U(T)) \otimes_{U(T)} C, d^{\prime}, d^{\prime \prime}\right\}, \\
A^{p, q} & =\left(\bigwedge^{-p} T \otimes U(T)\right) \otimes_{U(T)} C^{q}, \quad d^{\prime}=\delta \otimes_{U(T)} 1, d^{\prime \prime}=1 \otimes_{U(T)} d .
\end{aligned}
$$

Then we have the following two spectral sequences (cf. MacLane [9, XI. 6] and Cartan-Eilenberg [1, XIII. 2]) :

$$
\begin{aligned}
E_{2}^{p, q} & =\operatorname{Tor}_{U(T)}^{-p}\left(k, H^{q}\left(C^{*}\right)\right), \\
{ }^{p} E_{2}^{p, q} & =H^{q}\left(\operatorname{Tor}_{U(T)}{ }^{-p}\left(k, C^{*}\right)\right) .
\end{aligned}
$$

Consider the first spectral sequence. By the Cartan formula (1.2) the operation of $U(T)$ on $H^{*}(C)$ is trivial because for any cocycle 
$c$ and $X \in T, \quad \theta(X) c=\iota(X) d c+d \iota(X) c=d(\iota(X) c)$ is a coboundary. Hence we have

$$
{ }^{\prime} E_{2}^{p, q} \cong \operatorname{Tor}_{U(T)}^{-p}(k, k) \otimes H^{q}(C) \cong H_{-p}(T) \otimes H^{q}(C) .
$$

Note that $H_{\ell}(T)=0$ for $\ell<0$ or $\ell>n=\operatorname{dim} T$. Consider the second spectral sequence. Since each $C^{q}$ is a projective $U(T)$-module, this spectral sequence collapses and we obtain

$$
" E_{2} \cong H^{*}\left(k \otimes_{U(T)} C\right) .
$$
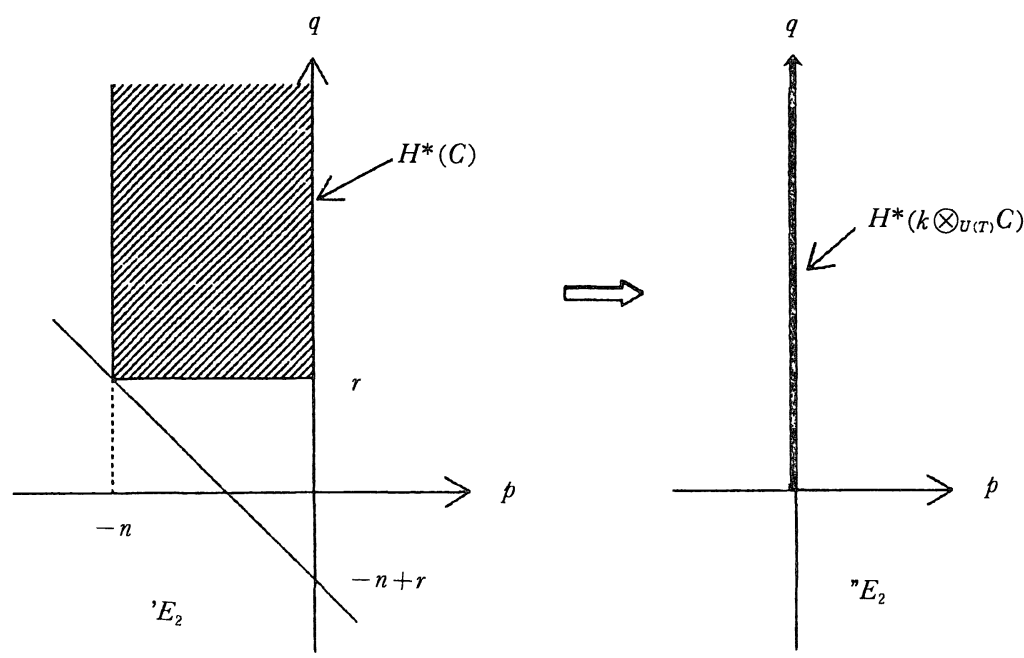

Figure $\mathbb{1}$

Therefore for the first spectral sequence,

$$
{ }^{\prime} E_{2}^{p, q} \Rightarrow H^{p+q}\left(k \otimes_{U(T)} C\right) \text {. }
$$

Let $r$ be the first integer such that $H^{r}(C) \neq 0$. Then

$$
\begin{aligned}
& ' E_{2}^{-n, r}=' E_{\infty}^{-n, r}=H^{r}(C) \neq 0, \\
& ' E_{2}^{-n-i, r+i}=' E_{2}^{-n+i, r-i}=0 .
\end{aligned}
$$

Consider $E_{\infty}$-term. Then we have ' $E_{\infty}^{-n, r} \cong H^{-n+r}\left(k \otimes_{U(T)} C\right)$. Since $H^{i}\left(k \otimes_{U(T)} C\right)=0$ for $i<0$, we obtain $-n+r \geqq 0$, i。 e, $r \geqq n$. Hence $H^{i}(C)=0$ for $i<n=\operatorname{dim} T$. This completes the proof.

Remark. If $C^{0}=0$, then $H^{i}\left(k \otimes_{U(T)} C\right)=0$ for $i \leqq 0$. Hence $H^{i}(C)$ $=0$ for $i \leqq n$. 


\section{§2. The Weil Algebra}

In this section we consider a spectral sequence associated to a filtration of the Weil algebra of $\mathfrak{A}_{n}$.

Let $k$ be a field of characteristic zero. Let $g$ be a Lie algebra and $\mathrm{g}^{*}$ a dual space of $\mathrm{g}$ with respect to a canonical topology.

Definition 2.1. The Weil algebra of a Lie algebra $\mathfrak{g}$, denoted by $W(\mathfrak{g})$, is $\wedge \mathfrak{g}^{*} \otimes S \mathfrak{g}^{*}$ as algebra, where the exterior algebra $\wedge \mathrm{g}^{*}$ is generated by 1 -forms $\alpha \in \mathrm{g}^{*}$, and the symmetric algebra $\mathrm{Sg}^{*}$ by 2 -forms $\Omega_{\alpha}$ for $\alpha \in \mathrm{g}^{*}$.

Its differential is defined by $d \alpha=d_{1} \alpha+\Omega_{\alpha}$, where $d_{1} \alpha \in \wedge^{2} \mathrm{~g}^{*}$ is the differential of $\alpha$ in the cochain complex of the Lie algebra $\mathfrak{g}$ with coefficients in $k$.

Its $\mathrm{g}$-operation is defined by making $\iota(X)$ (for $X \in \mathfrak{g}$ ) operate as the obvious anti-derivation on $\wedge \mathrm{g}^{*}$ and trivially on $\mathrm{Sg}^{*}$.

Consider the bidegree

$$
W^{2 p, q}(g)=\wedge^{q} g^{*} \otimes S^{p} g^{*} \quad \text { for } q, p>0 .
$$

Then we have a natural filtration $W=F^{0} \supset F^{1} \supset F^{2} \supset \cdots$, where $F^{s}=$ $\sum_{2 p \geq s} W^{2 p, *}$, which is compatible with the differential $d$.

The associated graded module is

$$
E_{0}^{s}(\mathfrak{g})=\left\{\begin{array}{cl}
\wedge^{*} \mathrm{~g}^{*} \otimes S^{p} \mathrm{~g}^{*} & \text { for } s=2 p, p=0,1,2, \cdots \\
0 & \text { otherwise }
\end{array}\right.
$$

By the calculation we have

$$
E_{1}^{s}(\mathfrak{g})=\left\{\begin{array}{cl}
H^{*}\left(\mathfrak{g}: S^{p}\left(\mathfrak{g}^{*}\right)\right) & \text { for } s=2 p, p=0,1,2, \cdots \\
0 & \text { otherwise. }
\end{array}\right.
$$

Now we consider the Lie algebra $a_{n}$ of formal vector fields on $\boldsymbol{R}^{n}$ and its maximal abelian subalgebra

$$
T=\left\{\lambda_{1} D_{1}+\cdots+\lambda_{n} D_{n} ; \lambda_{i} \in k\right\} \text { where } D_{i}=\partial / \partial x_{i} .
$$

Lemma 2.2. The continuous dual space $\mathfrak{a}_{n}^{*}$ and the $m$-th tensor product $\otimes{ }^{m} \mathfrak{a}_{n}^{*}(m>0)$ of $\mathfrak{a}_{n}^{*}$ are free $U(T)$-modules.

The proof of this lemma can be found in [12].

Since $\wedge^{q} \mathfrak{a}_{n}^{*} \otimes S^{p} \mathfrak{a}_{n}^{*}(q+p>0)$ is a direct summand of $\otimes^{4+p} \mathfrak{a}_{n}^{*}$, we 
get the following corollary:

Corollary 2. 3. $E_{0}^{2 p, q}\left(\mathfrak{a}_{n}\right)$ is a projective $U(T)$-module where $p+q>0$.

It is easy to check that the $a_{n}$ cochain algebra structure of $W\left(\mathfrak{a}_{n}\right)$ induces a $T$-cochain complex structure on $E_{0}^{2 p}\left(\mathfrak{a}_{n}\right)$. Hence from Proposition 1.3 and corollary 2.3 we have the following proposition :

\section{Proposition 2.4.}

$$
E_{1}^{2 p, q}\left(\mathfrak{a}_{n}\right)=H^{q}\left(\mathfrak{a}_{n} ; S^{p}\left(\mathfrak{a}_{n}^{*}\right)\right)=0 \text { for } p>0, q<n \text {. }
$$

Remark. Let $g$ be a finite dimensional reductive Lie algebra. The following fact is well-known:

$$
E_{1}^{2 *, q}(\mathfrak{g})=H^{q}(\mathfrak{g}) \otimes I^{*}(\mathfrak{g})
$$

where $I^{*}(\mathrm{~g})$ is the algebra of polynomials on $\mathrm{g}$ invariant under coadjoint operation. In the case of $\mathfrak{a}_{n}$, it seems that the following holds :

$$
E_{2}^{2 p, *}\left(\mathfrak{a}_{n}\right)=H^{*-2 n}(\mathfrak{g l}(n, \mathbb{R})) \otimes I^{p+n} \mathfrak{g}(\mathfrak{l}(n, \mathbb{R})) .
$$

When $p=1$, this is true (see [4]).

\section{§ 3. The Cohomology of Formal G-invariant Vector Fields}

In this section we recall the result obtained in [7] where a similar type of cohomology was studied.

First we recall a few facts about topological vector spaces over discrete fields, which are useful in studying infinite dimensional Lie algebras.

Let $\Delta$ be a topological field with the discrete topology. We say that a topological vector space $E$ over $\Delta$ is linearly compact when $E$ is a projective limit of finite dimensional discrete vector spaces.

Let $E$ be a topological vector spaces over $\Delta$, and $E^{*}$ the topological dual of $E$. We topologize $E^{*}$ by prescribing, for a system of neighborhoods of the origin, the collection of all sets of the form $F^{\perp}$, where $F$ is a linearly compact subspace of $E$ and $F^{\perp}$ is its annihilator in $E^{*}$. 
Let $E$ and $F$ be topological vector spaces. Consider the ordinary tensor product $E^{*} \otimes F^{*}$ and give it the discrete topology. We define the topological tensor product of $E$ and $F$ to be the space $\left(E^{*} \otimes F^{*}\right)^{*}$, which will be denoted by $E \otimes F$. We note that when $E$ and $F$ are linearly compact, so is $E \hat{\otimes} F$ (see [5]).

Now we recall a formal $G$-invariant vector fields. Let $\mathfrak{g}$ be a linearly compact Lie algebra; that is, a topological Lie algebra and linearly compact as a topological vector space. Consider the direct sum $\mathfrak{a}_{n} \oplus \boldsymbol{R}[[x]] \otimes \mathfrak{g}$, denoted by $\mathfrak{a}_{n, \text { a }}$, where $\boldsymbol{R}[[x]]$ is the ring of all formal power series in $n$-variables over $\boldsymbol{R}$ and linearly compact with respect to the Krull topology. From definition, the canonical action of $\mathfrak{a}_{n}$ on $R[[x]]$ induces the action of $\mathfrak{a}_{n}$ on $R[[x]] \otimes g$. We define the bracket operation as follows:

$$
\left[\left(X_{1}, H_{1}\right),\left(X_{2}, H_{2}\right)\right]=\left(\left[X_{1}, X_{2}\right], X_{1} H_{2}-X_{2} H_{1}-\left[H_{1}, H_{2}\right]\right)
$$

where $X_{i} \in \mathfrak{a}_{n}, H_{i} \in \boldsymbol{R}[[x]] \otimes \mathfrak{g}$ and the bracket $\left[H_{1}, H_{2}\right]$ is induced by the bracket operation $\mathfrak{g} \otimes \mathfrak{g} \rightarrow \mathfrak{g}$. Hence we may give $\mathfrak{a}_{n, s}$ the structure of linearly compact Lie algebra.

Before we state the fact concerning the cohomology of $\mathfrak{a}_{n, 8}$ we review a notation. Define:

$$
W_{n}(\mathrm{~g}) \cong W(\mathfrak{g}) / W \cdot S^{n+1}\left(\mathrm{~g}^{*}\right)
$$

where $W \cdot S^{n+1}\left(\mathrm{~g}^{*}\right)$ is the ideal of Weil algebra $W(\mathrm{~g})$ generated by the $(n+1)-t h$ symmetric product space $S^{n+1}\left(\mathfrak{g}^{*}\right)$. Let $\mathfrak{g l}_{n}$ be the Lie algebra of all $n \times n$ real matrices.

Let $\pi: \mathfrak{a}_{n, \mathfrak{s}} \longrightarrow \mathrm{gl}_{n} \oplus \mathrm{g}$ be the projection defined by

$$
\mathfrak{a}_{n} \ni \sum_{i=1}^{n}\left(a^{i}+a_{j}^{i} x^{j}+(\text { higher order })\right) \partial / \partial x^{i} \longrightarrow\left(-a_{\jmath}^{i}\right)_{i, j} \in \mathfrak{g} \mathfrak{l}_{n},
$$

and

$$
\varepsilon \otimes i d: R\left[\left[x_{1}, \cdots, x_{n}\right]\right] \otimes \mathfrak{g} \longrightarrow \boldsymbol{R} \otimes \mathrm{g}=\mathrm{g}
$$

where $\varepsilon$ is a canonical projection. Then $\pi$ induces a cochain map

$$
\Phi(\pi): W_{n}\left(\mathfrak{g l}_{n} \oplus \mathfrak{g}\right) \longrightarrow C^{*}\left(\mathfrak{a}_{n, \mathfrak{s}}\right)
$$

where $C^{*}\left(\mathfrak{a}_{n, \mathrm{~s}}\right)$ is a cochain complex of $\mathfrak{a}_{n, 8}$ with values in $\boldsymbol{R}$. (see Hamasaki [7, p. 408]).

Proposition 3. 1. If $\mathfrak{g}$ is a linearly compact Lie algebra, then $\pi$ induces an isomorphism 


$$
\Phi(\pi)^{*}: H^{*}\left(W_{n}\left(\mathfrak{g} \mathfrak{l}_{n} \oplus \mathfrak{g}\right)\right) \cong H^{*}\left(\mathfrak{a}_{n, 8}\right)
$$

Remark. In [7] this proposition was proved on condition that $\mathfrak{g}$ is a finite dimensional Lie algebra. But the finite dimensionality is not essential. We need the following two conditions:

i) $(\boldsymbol{R}[[x]] \otimes \hat{g})^{*} \cong \mathbb{R}[[x]]^{*} \otimes \mathrm{g}^{*}$,

ii) with respect to a basis $\left\{\omega_{i}\right\}_{i \in \mathrm{I}}$ of $\mathrm{g}^{*}$, there is a family $\left\{\xi_{i} \in\right.$ $\mathrm{g} ; i \in I\}$ such that $\omega_{\imath}\left(\xi_{j}\right)=1 \quad(i=j), 0$ (otherwise).

It is known that, when $E$ is linearly compact or discrete, $E \cong E^{* *}$ (see [5]). Since $\mathbb{R}[[x]]^{*} \otimes \mathrm{g}^{*}$ is discrete,

$$
(\mathbb{R}[[x]] \otimes \mathfrak{g})^{*} \cong\left(\left(\mathbb{R}[[x]]^{*} \otimes \mathfrak{g}^{*}\right)^{*}\right)^{*} \cong \mathbb{R}[[x]]^{*} \otimes \mathfrak{g}^{*}
$$

Since $\mathrm{g}^{*}$ is discrete, we can find $\left\{\xi_{i} \in \mathrm{g}^{* *} ; i \in I\right\}$ such that $\omega_{i}\left(\xi_{j}\right)$ $=1 \quad(i=j), 0$ (otherwise). Hence above two conditions are satisfied.

\section{\$4. The Main Theorem}

In this section we will state the main theorem and prove it.

Let $\iota: \mathfrak{a}_{r}=\mathbb{R}[[x]] \otimes \mathbb{R}^{r}-\ldots \rightarrow \mathfrak{a}_{r, n-r}=\mathbb{R}[[x]] \otimes \mathbb{R}^{r} \oplus \mathbb{R}[[x, y]] \otimes \mathbb{R}^{n-r}$ be the inclusion map to the first factor of the direct sum.

Theorem 4. 1. The inclusion map e induces an isomorphism of cohomology

$$
\iota^{*}: H^{i}\left(\mathfrak{a}_{r, n-r}\right) \cong H^{i}\left(\mathfrak{a}_{r}\right) \text { for } i \leqq n+r \text {. }
$$

First consider the Lie algebra $\mathfrak{a}_{r, a_{n-r}}=\mathfrak{a}_{r} \oplus \mathbb{R}[[x]] \otimes \mathfrak{a}_{n-r}(x=$ $\left.\left(x^{1}, \ldots, x^{r}\right)\right)$ defined in $\S 3$. Since $\mathbb{R}[[x]] \otimes \mathbb{R}[[y]]=\mathbb{R}[[x, y]](y=$ $\left.\left(y^{1}, \cdots, y^{n-r}\right)\right), \mathfrak{a}_{r_{0} \mathbb{a}_{n-r}}$ is isomorphic to $\mathfrak{a}_{r_{0}-r}$ Using this fact we consider the following commutative diagram concerning the canonical projections :

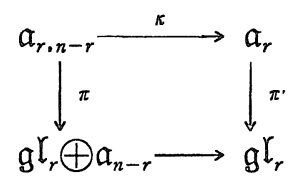

where $\kappa$ is a canonical projection which is a left inverse of $\iota$ and $\pi$, $\pi$ ' are projection introduced in $\S 3$. By the naturality of construction we obtain the following commutative diagram: 


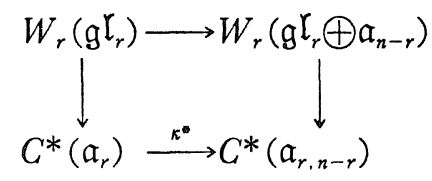

where $C^{*}(\mathfrak{a})$ is the continuous cohomology of $\mathfrak{a}$. On the other hand by the Proposition 3.1, we have:

Lemma 4.2.

$$
H^{*}\left(\mathfrak{a}_{r, n-r}\right) \cong H^{*}\left(W_{r}\left(\mathfrak{g} \mathfrak{l}_{r} \oplus \mathfrak{a}_{n-r}\right)\right)
$$

Taking cohomology of the above diagram we have the following commutative diagram:

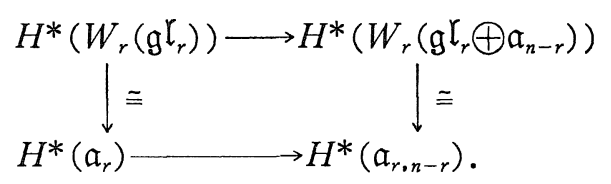

Hence in order to prove the theorem we consider the relation between the truncated Weil algebras $W_{r}\left(\mathfrak{g l}_{r}\right)$ and $W_{r}\left(\mathfrak{g l}_{r} \oplus \mathfrak{a}_{n-r}\right)$.

Note that $W\left(\mathfrak{g} \mathfrak{l}_{r} \oplus \mathfrak{a}_{n-r}\right) \cong \wedge^{*}\left(\mathfrak{g} \mathfrak{l}_{r} \oplus \mathfrak{a}_{n-r}\right) * \otimes S^{*}\left(\mathfrak{g} \mathfrak{l}_{r} \oplus \mathfrak{a}_{n-r}\right) * \cong \wedge^{*} \mathfrak{g} \mathfrak{l}_{r}^{*} \otimes$ $S^{*} \mathfrak{g l}_{r}^{*} \otimes \wedge^{*} \mathfrak{a}_{n-r}^{*} \otimes S^{*} \mathfrak{a}_{n-r}^{*} \cong W\left(\mathfrak{g l}_{r}\right) \otimes W\left(\mathfrak{a}_{n-r}\right)$. Since the ideal in $W\left(\mathfrak{g l}_{r} \oplus\right.$ $\left.\mathfrak{a}_{n-r}\right)$ generated by $S^{r+1}\left(\mathfrak{g l}_{r}^{*}\right)$ is contained in the ideal generated by $S^{r+1}\left(\mathfrak{g l}_{r} \oplus \mathfrak{a}_{n-r}\right) *$, we have a canonical homomorphism and a commutative diagram

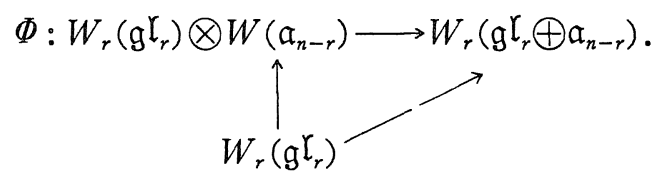

Lemma 4.4.

$$
\Phi^{*}: H^{i}\left(W_{r}\left(\mathfrak{g} \mathfrak{l}_{r}\right) \otimes W\left(\mathfrak{a}_{n-r}\right)\right) \longrightarrow H^{i}\left(W_{r}\left(\mathfrak{g} \mathfrak{l}_{r} \oplus \mathfrak{a}_{n-r}\right)\right)
$$

is an isomorphism for $i \leqq n+r$.

Proof. The truncated Weil algebra $W_{r}\left(\mathfrak{g l}_{r}\right) \otimes W\left(\mathfrak{a}_{n-r}\right)$ has a natural filtration induced by that of the Weil algebra $W\left(\mathfrak{g} \mathfrak{l}_{r} \oplus \mathfrak{a}_{n-r}\right)$. By the calculation we have

$$
\begin{aligned}
& E_{2}^{2 p+1, q}=0, \\
& E_{2}^{2 p, q}=\sum_{\imath \in z_{,} ! r_{r}} H^{i}\left(\mathfrak{g l}_{r} ; S^{\jmath}\left(\mathfrak{g l}_{r}\right)\right) \otimes H^{q-i}\left(\mathfrak{a}_{n-r} ; S^{p-j}\left(\mathfrak{a}_{n-r}\right)\right) .
\end{aligned}
$$


Consider the case that $p>r$ and $q<n-r$. Then $p-j \geqq p-r>0$ and $q-i<n-r$ or $i<0$. From Proposition 2.3, in this case

$$
E_{2}^{2 p, q}=0 \text { for } p>r \text { and } q<n-r \text {. }
$$

On the other hand the truncated Weil algebra $W_{r}\left(\mathfrak{g} \mathfrak{l}_{r} \oplus \mathfrak{a}_{n-r}\right)$ also has a natural filtration. The $E_{2}$-term of the corresponding spectral sequence is

$$
\left\{\begin{array}{l}
E_{2}^{2 p_{0} q}=\sum_{i, j \in z} H^{i}\left(g \mathfrak{l}_{r} ; S^{j}\left(g \mathfrak{l}_{r}\right)\right) \otimes H^{q-i}\left(\mathfrak{a}_{n-r} ; S^{p-j}\left(\mathfrak{a}_{n-r}\right)\right) \\
E_{2}^{p, q}=0 \quad \text { when } p \text { is odd or } p>2 r .
\end{array}\right.
$$

By the construction, $\Phi$ preserves the filtrations. Hence we have a homomorphism of spectral sequences where

$$
\begin{aligned}
& \Phi:(E, d) \longrightarrow\left(' E,{ }^{\prime} d\right), \\
& E \Rightarrow H\left(W_{r}\left(\mathfrak{g l}_{r}\right) \otimes W\left(\mathfrak{a}_{n-r}\right)\right) \\
& { }^{\prime} E \Rightarrow H\left(W_{r}\left(\mathfrak{g l} \mathfrak{l}_{r} \oplus \mathfrak{a}_{n-r}\right)\right) .
\end{aligned}
$$

Note that

$$
E_{2 k-1} \cong E_{2 k} \text { and ' } E_{2 k-1} \cong ' E_{2 k} \text { for } k \geqq 1 \text {. }
$$

In order to prove the lemma, we shall show the following by induction :

$$
\left(*_{k}\right) \Phi_{2 k}^{p, q}: E_{2 k}^{p, q} \longrightarrow E_{2 k}^{p \cdot q}
$$

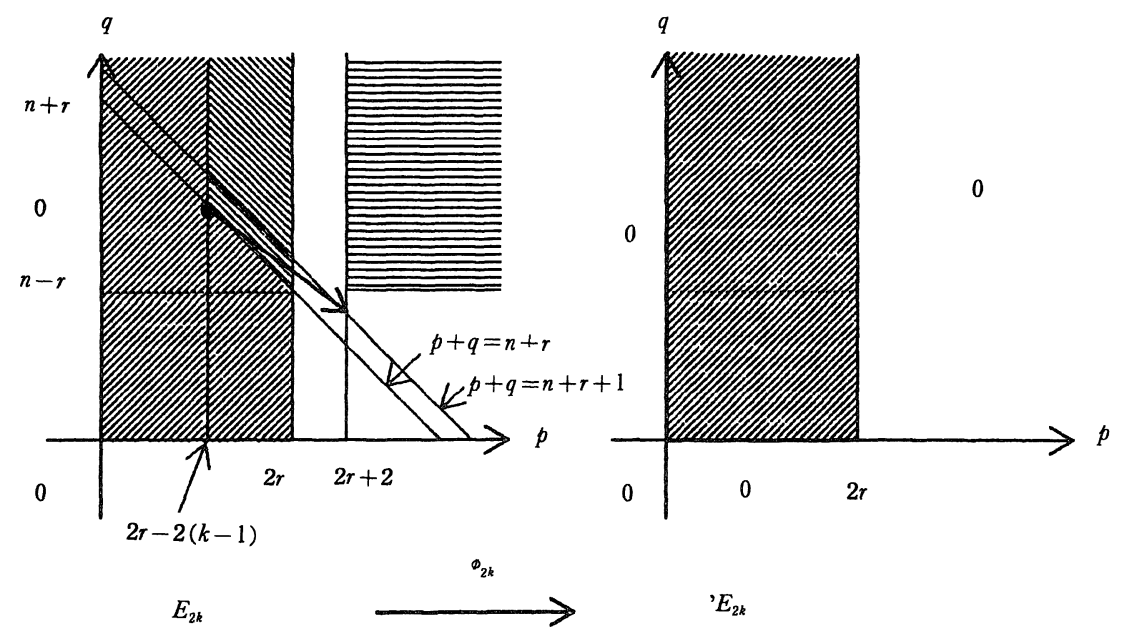

Figure 2 
is a monomorphism for $p \leqq 2 r$,

and an isomorphism for $p \leqq 2 r-2(k-1)$ or $p+q \leqq n+r$.

When $k=1, \Phi_{2}^{p . q}$ is an identity map for $p \leqq 2 r$ or $p+q \leqq n+r$. Assume that $\left(*_{k}\right)$ holds. Consider the first case i. e. $p \leqq 2 r$. Then $\Phi_{2 k}^{p, q}$ is a monomorphism, and $\Phi_{2 k}^{p-2 k, q+2 k-1}$ is an isomorphism because $p-2 k \leqq 2 r$ $-2(k-1)$.

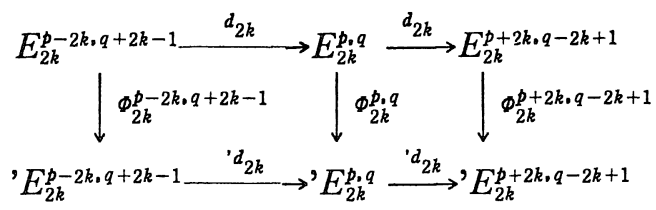

By diagram chasing and considering (4.5) we can see that

$$
\Phi_{2(k+1)}^{p, q}: E_{2(k+1)}^{p, q} \longrightarrow E_{2(k+1)}^{p, q}
$$

is a monomorphism for $p \leqq 2 r$. Next consider the second case $p \leqq$ $2 \mathrm{r}-2\{(k+1)-1\}$. Then $\Phi_{2 k}^{p \cdot q}$ and $\Phi_{2 k}^{p-2 k \cdot q+2 k-1}$ are isomorphisms, and $\Phi_{2 k}^{p+2 k, q-2 k+1}$ is monomorphism because $p+2 k \leqq 2 r$. By diagram chasing and considering (4.5) we can see that $\Phi_{2(k+1)}^{p, q}$ is an isomorphism for $b \leqq 2 r-2\{(k+1)-1\}$. Next consider the last case, i. e. $p+q \leqq n+r$. Then $\Phi_{2 k}^{p, q}$ and $\Phi_{2 k}^{p-2 k, q+2 k-1}$ are isomorphisms. If $p+2 k>2 r$ and $q-2 k+1$ $<n-r$, then $E_{2 k}^{p+2 k, q-2 k+1} \cong{ }^{\prime} E_{2 k}^{p+2 k, q-2 k+1} \cong 0$. Hence $\Phi_{2 k}^{p+2 k, q-2 k+1}$ is monomorphism. When $q-2 k+1 \geqq n-r$, considering $p+q \leqq n+r$, we have

$$
p+2 k+n-r \leqq p+2 k+q-2 k+1 \leqq n+r+1 .
$$

Hence $p+2 k \leqq 2 r+1$. Since $E_{2 k}^{2 r+1, *} \cong E_{2 k}^{2 r+1, *} \cong 0$ and $\left({ }_{k}\right), \Phi_{2 k}^{p+2 k, q-2 k+1}$ is monomorphism. In the same way as the second case we see that $\Phi_{i(k+1)}^{p \cdot q}$ is an isomorphism for $p+q \leqq n+r$. This proves $\left(*_{k+1}\right)$. Hence $\left(*_{k}\right)$ holds for any positive integer $k$. Using this, now we prove the Lemma. Consider the $E_{\infty}$-term. Then we see that

$$
\Phi_{\infty}^{p, q}: E_{\infty}^{p, q} \longrightarrow E_{\infty}^{p, q}
$$

is an isomorphism for $p+q \leqq n+r$. Since each spectral sequence converges, we see that

$$
\Phi^{*}: H^{i}\left(W_{r}\left(\mathfrak{g l}_{r}\right) \otimes W\left(\mathfrak{a}_{n-r}\right)\right) \longrightarrow H^{i}\left(W_{r}\left(\mathfrak{g l}_{r} \oplus \mathfrak{a}_{n-r}\right)\right)
$$

is an isomorphism for $i \leqq n+r$. This completes the proof of Lemma 4. 4 .

It is well-known that the Weil algebra is acyclic (cf. Natsume 
[10]). By the Kunneth formula, we have

$$
H^{i}\left(W_{r}\left(\mathfrak{g} \mathfrak{l}_{r}\right) \otimes W\left(\mathfrak{a}_{n-r}\right)\right) \cong H^{\imath}\left(W_{r}\left(\mathfrak{g} \mathfrak{l}_{r}\right)\right) \text {. }
$$

By the diagram (4.3) we obtain

$$
H^{i}\left(\mathfrak{a}_{r}\right) \cong H^{i}\left(\mathfrak{a}_{r, n-r}\right) \quad \text { for } i \leqq n+r \text {. }
$$

This completes the proof of the main Theorem 4.1 .

\section{§5. Some Remarks}

In this section we shall give a result and a conjecture concerning the Weil algebra of an infinite dimensional Lie algebra.

The structure of the Weil algebra $W(\mathrm{~g})$ of a finite dimensional reductive Lie algebra $\mathrm{g}$ is almost completely determined. By contraries there seems to be no study of the infinite dimensional case but [10] where the cohomology $H^{*}\left(W\left(\mathfrak{a}_{n}\right), \mathrm{gl}_{n}\right)$ of $\mathrm{gl}_{n}$-basic subcochain algebra of $W\left(\mathfrak{a}_{n}\right)$ was determined by calculating the spectral sequences. The result is

$$
H^{*}\left(W\left(\mathfrak{a}_{n}\right), \mathfrak{g} \mathfrak{l}_{n}\right) \cong I^{*}\left(\mathfrak{g} \mathfrak{l}_{n}\right)
$$

where $I^{*}\left(\mathfrak{g} \mathfrak{l}_{n}\right)$ is the algebra of polynomials on $\mathfrak{g}$ invariant under coadjoint operation by $g$. Note that we can deduce this result using the following Theorem (see Kamber and Tondeur [8, Theorem 5.64]) :

Theorem 5.1. Let $(\mathfrak{g}, \mathfrak{h})$ be a reduced pair of Lie algebras and $\theta$ : $\mathfrak{g} \rightarrow \mathfrak{h}$ an equivariant splitting of the exact $h$-module sequence $0 \rightarrow \mathfrak{h} \rightarrow \mathfrak{g} \rightarrow \mathfrak{g}$ / $\mathfrak{h} \rightarrow 0$. Let $K(\theta): w(\mathfrak{G}) \rightarrow w(\mathfrak{g})$ be the Weil homomorphism. Then the induced map on $\mathfrak{h}$-basic elements

$$
K(\theta)_{\mathfrak{G}}: I(\mathfrak{h}) \rightarrow W(\mathfrak{g})_{\mathfrak{G}-\text { basic }}
$$

is a homotopy equivalence.

Since the pair $\left(\mathfrak{a}_{n}, \mathfrak{g l} \mathfrak{l}_{n}\right)$ satisfies above conditions (see [7]), we have

$$
K(\theta)_{\mathrm{gl}_{n}}^{*}: I^{*}\left(\mathfrak{g} \mathfrak{l}_{n}\right) \cong H^{*}\left(W\left(\mathfrak{a}_{n}\right), \mathfrak{g l} \mathfrak{l}_{n}\right)
$$

We can also apply the above theorem to the pair $\left(H_{2 n}, \mathfrak{S p}_{2 n}\right)$ where $H_{2 n}$ is the Lie algebra of Hamiltonian vector fields on $\mathbb{R}^{2 n}$ and $\mathfrak{S p}_{2 n}$ 
is the Lie algebra of the Symplectic group.

Even if the total cohomology is calculated, we are rather interested in the $E_{2}$-term of the spectral sequence converging to $H^{*}$ $\left(W\left(\mathfrak{a}_{n}\right), \mathfrak{g l}_{n}\right)$. Consider the filtration of $W\left(\mathfrak{a}_{n}\right)_{\mathfrak{a r}_{n}-\text { basic }}$ induced by the one of $W\left(a_{n}\right)$ studied in $\S 2$. By the similar calculation we have

$$
E_{2}^{p, 1} \cong H^{*}\left(\mathfrak{a}_{n, 8 l_{n}} ; S^{p}\left(\mathfrak{a}_{n}\right)\right) \text {. }
$$

In [3] and [4], the following was calculated:

$$
\begin{aligned}
& E_{2}^{0, *} \cong H^{*}\left(\mathfrak{a}_{n, 8 \mathfrak{I}_{n}} ; \boldsymbol{R}\right) \cong I^{*}\left(\mathfrak{g l}_{n}\right) / \operatorname{deg}>2 n, \\
& E_{2}^{2, q} \cong H^{q}\left(\mathfrak{a}_{n, 8 \mathfrak{s I}_{n}} ; \mathfrak{a}_{n}^{*}\right) \cong \begin{cases}I^{n+1}\left(\mathfrak{g l}_{n}\right) & q=2 n, \\
0 & \text { otherwise. }\end{cases}
\end{aligned}
$$

Using Proposition 2.3 we can easily see that

$$
E_{2}^{2 p, q} \cong H^{q}\left(\mathfrak{a}_{n}, \mathfrak{g}_{n} ; S^{p}\left(\mathfrak{a}_{n}\right)\right) \cong 0 \quad \text { for } p>0, q<n .
$$

The vast range remains unknown. But studying the Theorem 5.1 it seems that $I^{n+i}=E_{2}^{2 n 22 n}$.

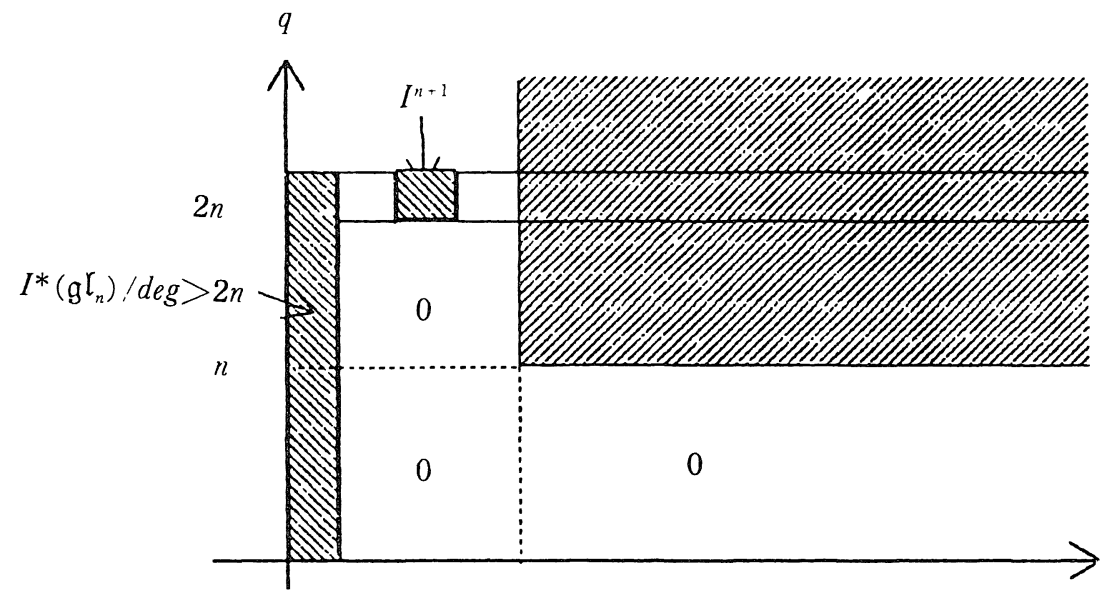

Figure 3

\section{References}

[1] Cartan, H. and Eilenberg, S., Homological algebra, Princeton Univ. Press, 1956.

[2] Feigin, B. L., Characteristic classes of flags of foliations, Funkt. Analiz $i$ Ego Prilozhen., vol. 9, no. 4(1975), 49-56.

[3] Gel'fand, I. M. and Fuks, D. B., The cohomology of the Lie algebra of formal vector 
fields, Izv. Acad. Nauk SSSR, 34 (1970), 327-342.

[4] Gel'fand, I. M., Feigin, B. L. and Fuks, D. B., Cohomologies of Lie algebras of formal vector fields with coefficient, in their adjoint spaces, and variations of characteristic classes of foliations, Funkt. Analiz i Ego Prilozhen., 8 (1974), 13-30.

[5] Guillemin, V.W., A Jordan-Hoelder decomposition for a certain class of infinite dimensional Lie algebras, J. Differential Geometry', 2 (1968), 313-345.

[6] Guillemin, V.W. and Shnider, S., Some stable results on the cohomology of classical infinite dimensional Lie algebras, Trans. Am. Math. Soc., 179 (1973), 275-280.

[7] Hamasaki, A., Continuous cohomologies of Lie algebras of formal $G$-invariant vector fields and obstructions to lifting foliations, Publ. RIMS, Kyoto Univ., 20 (1984), 401429.

[8] Kamber, F. and Tondeur, P., Foliated bundles and characteristic classes, Lecture Notes in Math., 493 (1975), Springer-Verlag.

[9] MacLane, S., Homology, Springer, 1963.

[10] Natsume, T., Certain Weil algebras of infinite-dimensional Lie algebras, Kodai Math. J., 1 (1978), 401-410.

[11] Roger, C., Sur la cohomologie de l'algebre de Lie des champs de vecteurs de contact formels(II), C. R. Acad. Sci., Paris, 292 (1981), 893-896.

[12] Sithananthan, K., On the cohomology of the Lie algebra of formal vector fields preserving a flag, Illinois J. of Math., 28 (1984), 487-497.

[13] Vey, J., Sur la cohomologie des champs de vecteurs symplèctiques formels, C. $R$. Acad. Sci., Paris, 280A (1975), 805. 